\title{
A continuum model of drag and lift forces for inclined planes dragged through granular beds
}

\author{
Hong Guo ${ }^{1, *}$, Jiangtao Fu ${ }^{2}$, Rui Guo, Hong Jiang ${ }^{1}$ and Nengyuan Chen ${ }^{2}$ \\ 1 School of Civil Engineering and Architecture, Shaanxi University of Technology, Hanzhong 723000, China \\ 2 China electronic research institute of engineering investigations and design, $\mathrm{Xi}^{\prime}$ an 710054, China \\ * Correspondence: hguo@snut.edu.cn; Tel.:+86-15336204368
}

\begin{abstract}
Both drag and lift forces impact an inclined plane when it is dragged through a granular bed. In this paper, the following results have been obtained: the drag and lift forces grow with the velocity of motion; when the immersion depth is constant, the inclination angle has no effect on drag force, however, the lift force increases linearly with this inclination angle; the ratio of drag and lift forces is exactly equal to the tangent value of the inclined angle. In order to describe this physical process macroscopically, a continuum wedge model based on the Coulomb model is established to predict drag and lift forces. Particularly, the dynamic friction angle in the assumed shear band is predicted as a function of both inclined angle and moving velocity.
\end{abstract}

Keywords: granular flow; drag and lift forces; discrete element method

\section{Introduction}

Much of the matter on the surface of the earth is granular, such as sand, soil, snow etc. Granular materials can be found in geotechnical, industrial and agricultural processes. Activities such as mixing, plowing, skiing, and excavation all involve motion through a granular material[1]. Drag force is the main focus of rigid bodies dragged through granular media. We have already investigated the drag force on vertical plates[2], however, an inclined plate also experiences a lift force when it is dragged through a granular material, making the mechanical properties more complicated. Figure 1(a) shows a vertical plate, of width Land immersion depth $Z$, moving horizontally through granular matter that has volumetric density $\rho^{*}$. A wedge is created that slides along the shear band, which resembles a mass sliding along an inclined plane as shown in figure 1(b). In addition to the drag force $F_{d}$, the forces acting on the wedge are gravity, $m g$, the normal force, $N$, and the frictional resistance, $\mu N$, see figure 1(c). The pushed wall is a inclined plate with a width of $L$, as shown in figure $1(\mathrm{~d})$. Figure 1(e) shows all the forces applied on the wedge.

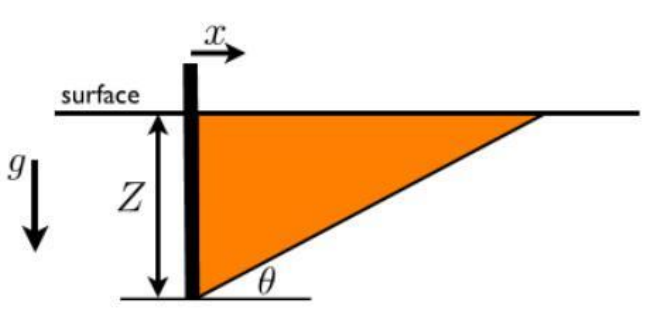

figure 1(a)

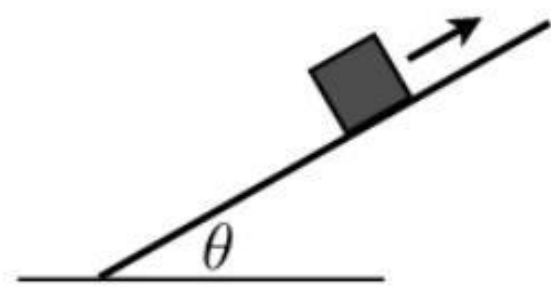

figure $1(b)$ 


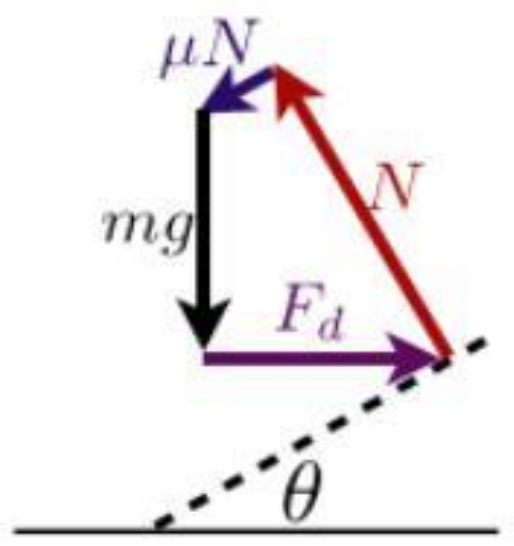

figure $1(\mathrm{c})$

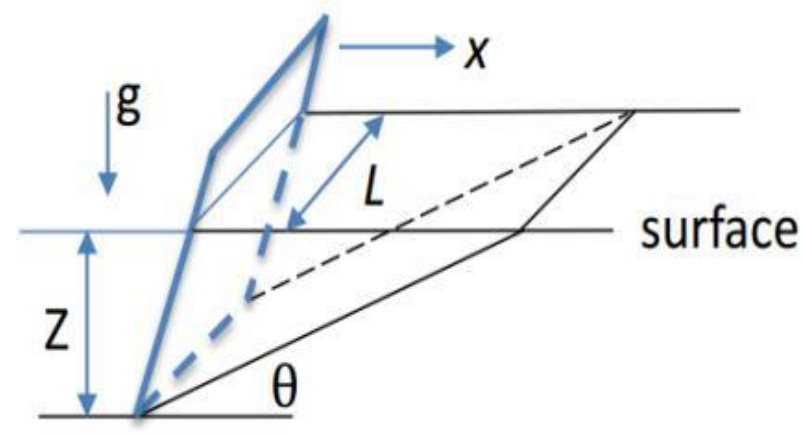

figure $1(\mathrm{~d})$

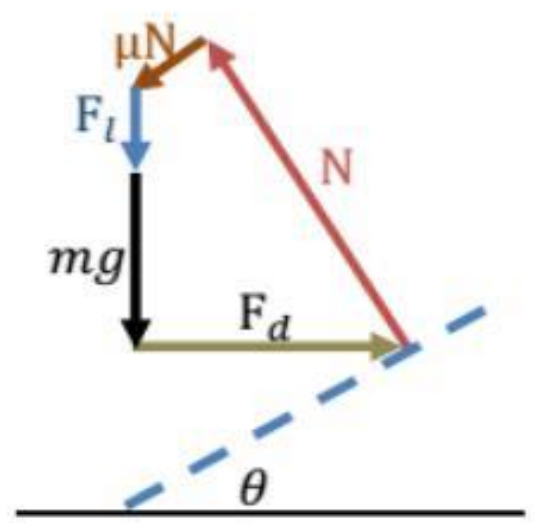

figure $1(\mathrm{e})$

Figure 1 I Schematic showing Coulomb's method of wedges

The drag force applied on the vertical plate[2] can be obtained by extending Coulomb's model, in which a passive yielding force can be determined through limit equilibrium, and can be described as

$$
F=m g \frac{\sin \theta+\mu \cos \theta}{\cos \theta-\mu \sin \theta}
$$

where $\theta$ is the angle of inclination, $\mu$ is the frictional coefficient and $m=\frac{1}{2} \rho^{*} Z^{2} L / \tan \theta$ is the mass of the wedge[3]. When passive yielding occurs within the granular matter in front of the advancing wall, the Coulomb model indicates that the wedge inclination angle is related to the internal frictional angle $(\phi): \theta_{0}=\frac{\pi}{4}-\frac{\phi}{2}$.

However, the influence of the plow speed on drag and lift forces acting on the inclined plate is still poorly understood. Although the Coulomb model takes the inclined retaining wall into account, the influence of the velocity and inclination angle still requires investigation. Recent work by Baptiste 
Percier et al.[4] dealing with an incline plow moving over a granular bed illustrates that drag and lift forces are not directly dependent on the velocity, but are proportional to the weight of the wedge that is formed from the pile-up in front of the plate. However, in this paper, we consider a more complicated geometry, in which both the position and direction of the shear band are varied. This makes the frictional properties complicated. Through the Discrete Element Simulation we find that drag and lift forces are very sensitive to the plow speed. We examine the total force applied on the inclined plate moving over large distances in granular beds through the Discrete Element Method. Inclined plates can induce both drag and lift forces. We further develop Coulomb's model, using the drag velocity and inclined angle as variables. As far as we know, this is the first attempt to apply Coulomb's model to the case where the velocity and inclination angle are varied.

One of the applications of this plate-induced granular flow is to washboards or corrugated roads caused by vehicles moving through granular material such as sand or soil[4-8]. In this case, the rolling wheel is simplified as an inclined plate[5,8]. The aim of the present paper is to derive a simple model for the drag and lift forces acting on the plate, accounting for the velocity and the inclined angle, the latter of which is a proxy for the wheel diameter. The drag speed is one of the controlling variables in washboard dynamics[9], which is the reason we focus one the velocity effect in the present paper. Figure 2 shows a wheel on a sandy road and its simplified model. Although there were some results using the Discrete Element Method[10,11], the influence of both drag velocity and angle of attack (inclined angle) is have not yet been investigated in detail.

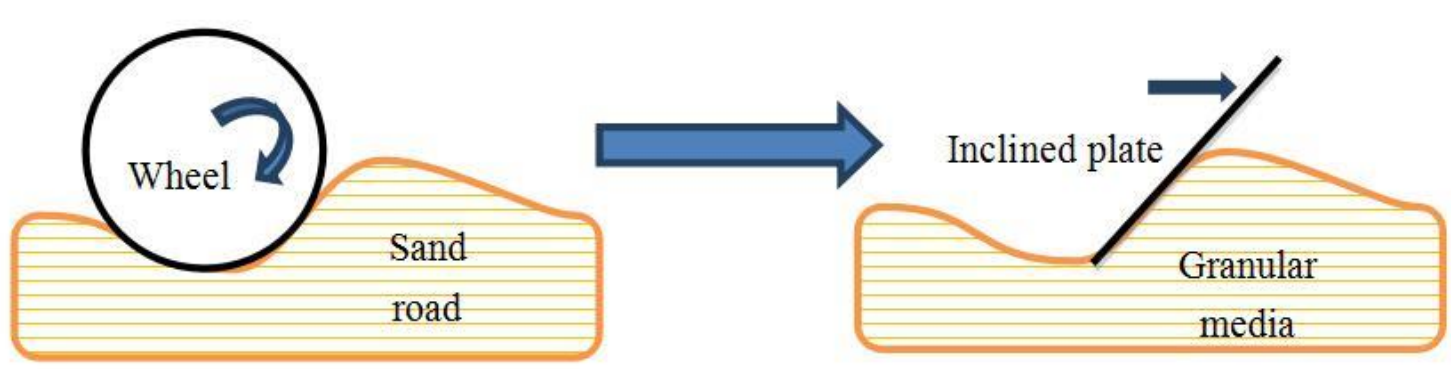

Figure 2 | Wheel effect and its simplified model

\section{Method}

Based on our work in reference[2], we investigate the effects of drag velocity and angle of inclination on drag and lift forces through the Two-dimensional Discrete Element approach. Figure 3(a) shows the normal contact model, in which $c_{n}$ is the normal damping constant, $c_{n}=2 \beta \sqrt{m^{*} K_{n}}$, where $m^{*}=$ $\frac{m_{1}+m_{2}}{2}$ is the average mass of the two contacted particles, and $K_{n}$ is the normal stiffness. Figure 3(b) shows the tangential contact model, in which $K_{s}$ is the tangential stiffness, and $\mu_{p}$ is the interparticle friction coefficient.

Hence, the normal and tangential contact forces can be described as

$$
\left\{\begin{array}{c}
F_{n}=K_{n} \delta_{n}-2 \beta \sqrt{m K_{n}} \delta_{n} \\
F_{s}=K_{s} \delta_{s} \leq \mu_{p} F_{n}
\end{array}\right.
$$


where $\delta_{n}$ and $\delta_{s}$ are the normal and tangential deformations, respectively, $\dot{\delta_{n}}$ is the normal deformation rate, and $m$ is the particle mass. The time step is $6.2 \times 10^{-5} \mathrm{~s}$ (which is $7 \%$ of the critical time step $\left.t_{\text {critical }}=\frac{2 \sqrt{m / k}}{\pi}\right)$.

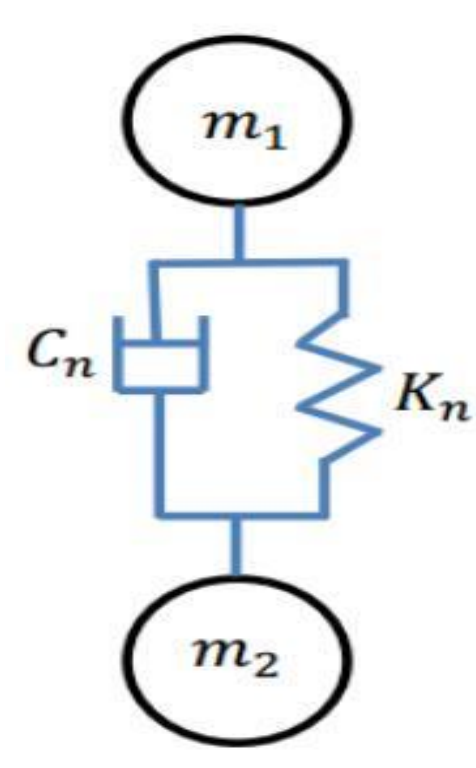

figure 3(a)

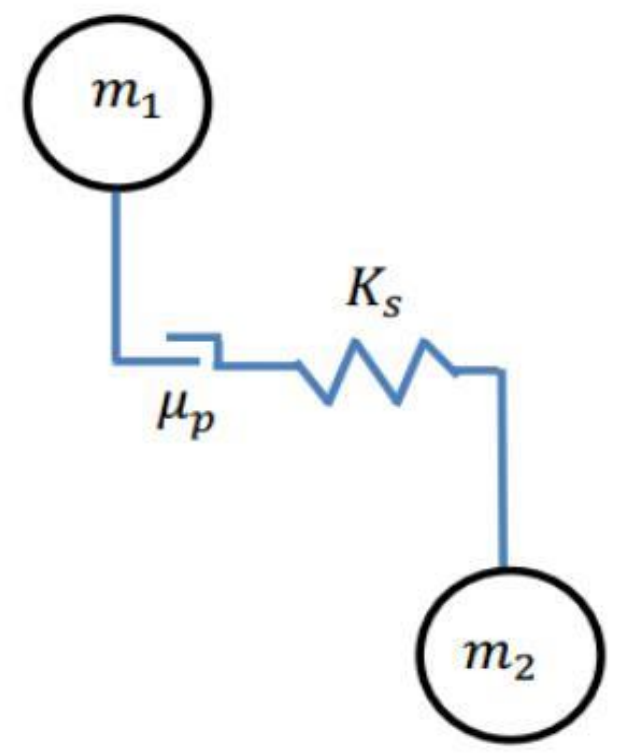

figure 3(b)

Figure 3 I Contact model in (a) normal and (b) tangential directions

The DEM model is shown as figure 4(a), and its flow field is illustrated in figure 4(b). The container's height is $0.3 \mathrm{~m}$, its width is $0.6 \mathrm{~m}$, and its immersion depth is $0.08 \mathrm{~m}$. The inclined angle $A$ is set to $30^{\circ}, 45^{\circ}$ and $60^{\circ}$, and the velocity $V$ is set to $0.2 \mathrm{~m} / \mathrm{s}, 0.1 \mathrm{~m} / \mathrm{s}$ and $0.02 \mathrm{~m} / \mathrm{s}$. All the particles in this paper are represented by cylinders with lengths of 1 meter. The radii of the cylinders are set to $5 \mathrm{~mm}$ with $20 \%$ random polydispersity, with a density of $2550 \mathrm{~kg} / \mathrm{m}^{3}$, a packing fraction of 0.84 . The microscopic friction coefficient (inter-particle friction coefficient $\mu_{d}$ ) between particles is 0.46 , the stiffness is $1 \times 10^{5}$ $\mathrm{N} / \mathrm{m}$, and the stiffness of the rigid body is 10 times larger than that of particles[2]. Since the particles during drag always suffer from elastic deformation, we use a simple linear elastic particle-particle contact model. However, the particles suffer energy dissipation, so we consider a normal contact damping that is confirmed by our free fall test based on reference[12]. From the free fall test, the coefficient of restitution can be obtained, and the relation between the coefficient of restitution $c_{R}$ and the damping ratio $\beta$ can be described as $\beta=f\left(c_{R}\right)$. 


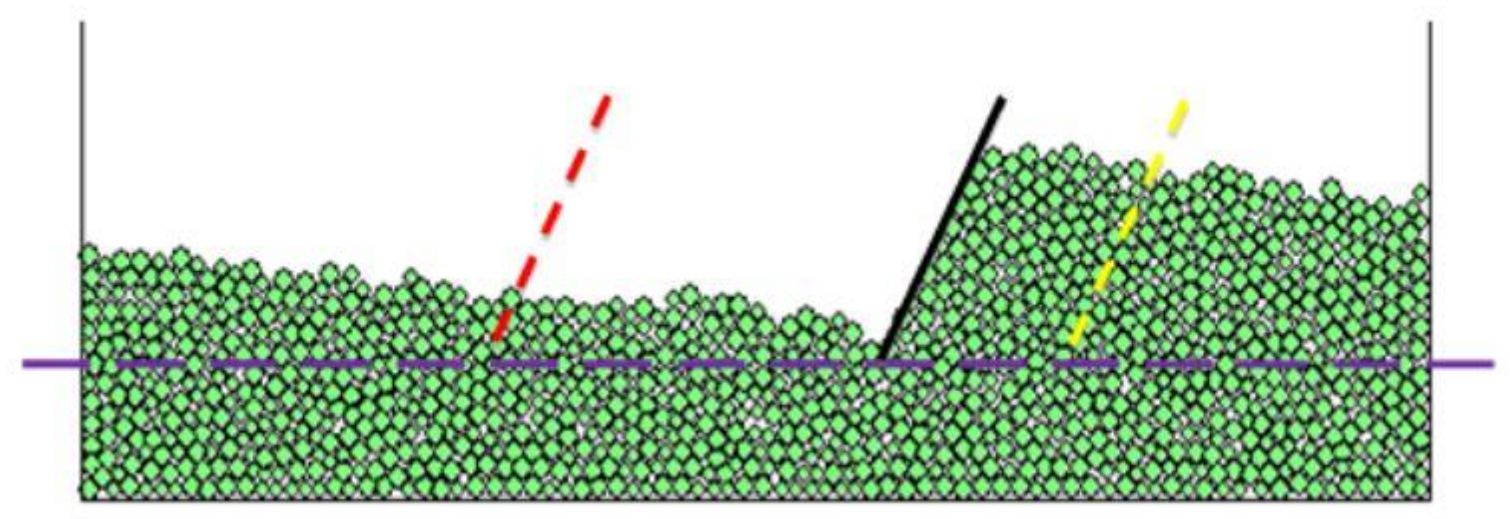

figure $4(\mathrm{a})$

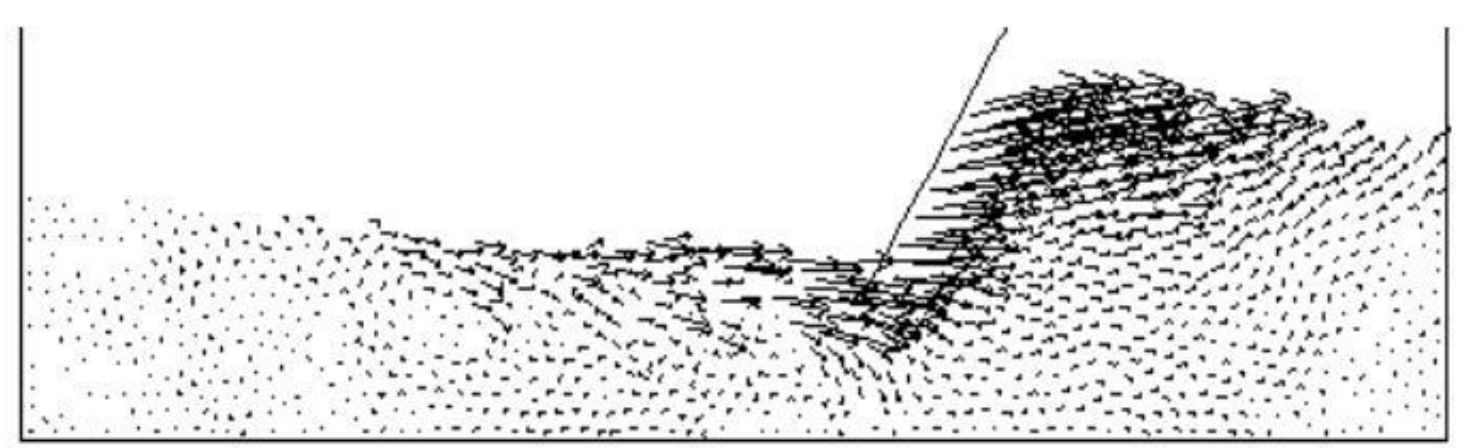

figure $4(b)$

Figure 4 I DEM model of the inclined plate dragged through granular media

Our study also shows that the microscopic parameters such as the inter-particle friction and stiffness have little influence on drag and lift forces if there is no friction between the rigid body and the particles (not detailed in this paper). The friction between the rigid body and particles is not taken into account in this paper. The reason is that we focus on the frictional effects among the particles themselves. We are more interested in the drag and lift forces that are caused by the flow of particles rather than the interaction of friction from the rigid body itself. This can be exactly controlled by DEM simulation, which is why we only use DEM tools here.

In figure 4(a), the rigid body is configured at a certain immersion depth and angle initially denoted by the purple and red dashed lines, respectively. Then the rigid body moves horizontally towards the ending position (represented by the yellow dashed line) under a constant velocity. In order to avoid boundary effects, the distance from the initial position to the left boundary and the distance from the ending position to the right boundary are all more than $10 \mathrm{~d}$. Every effective value is obtained by averaging 50 different numerical tests. The displacement field during the drag can be seen from figure $4(\mathrm{~b})$, where the lengths of the arrows reflect the displacement magnitudes. 


\section{Results}

Figure 5 illustrates the effect of velocity on drag and lift forces when the inclination angle is $60^{\circ}$. It is obvious that both the drag and lift forces increase with the drag velocity. A negative value of the drag force indicates that its direction is opposite to the velocity. Although there is boundary effect where the drag force increases dramatically near the final boundary[13], we do not consider this effect in this paper because we focus on effects far from the boundary. Actually, we restrict the total displacement to a reasonable range so that boundary effects can be avoided.

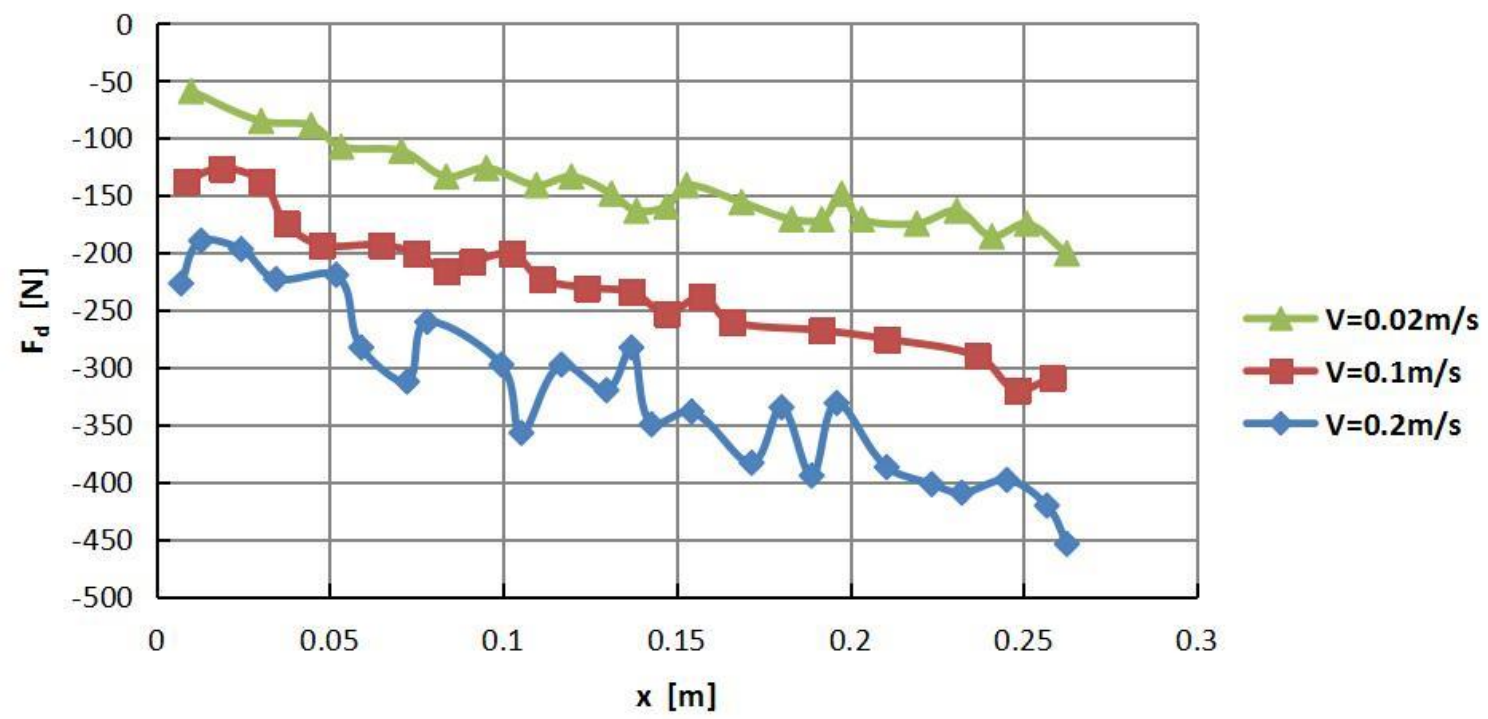

Figure 5(a)

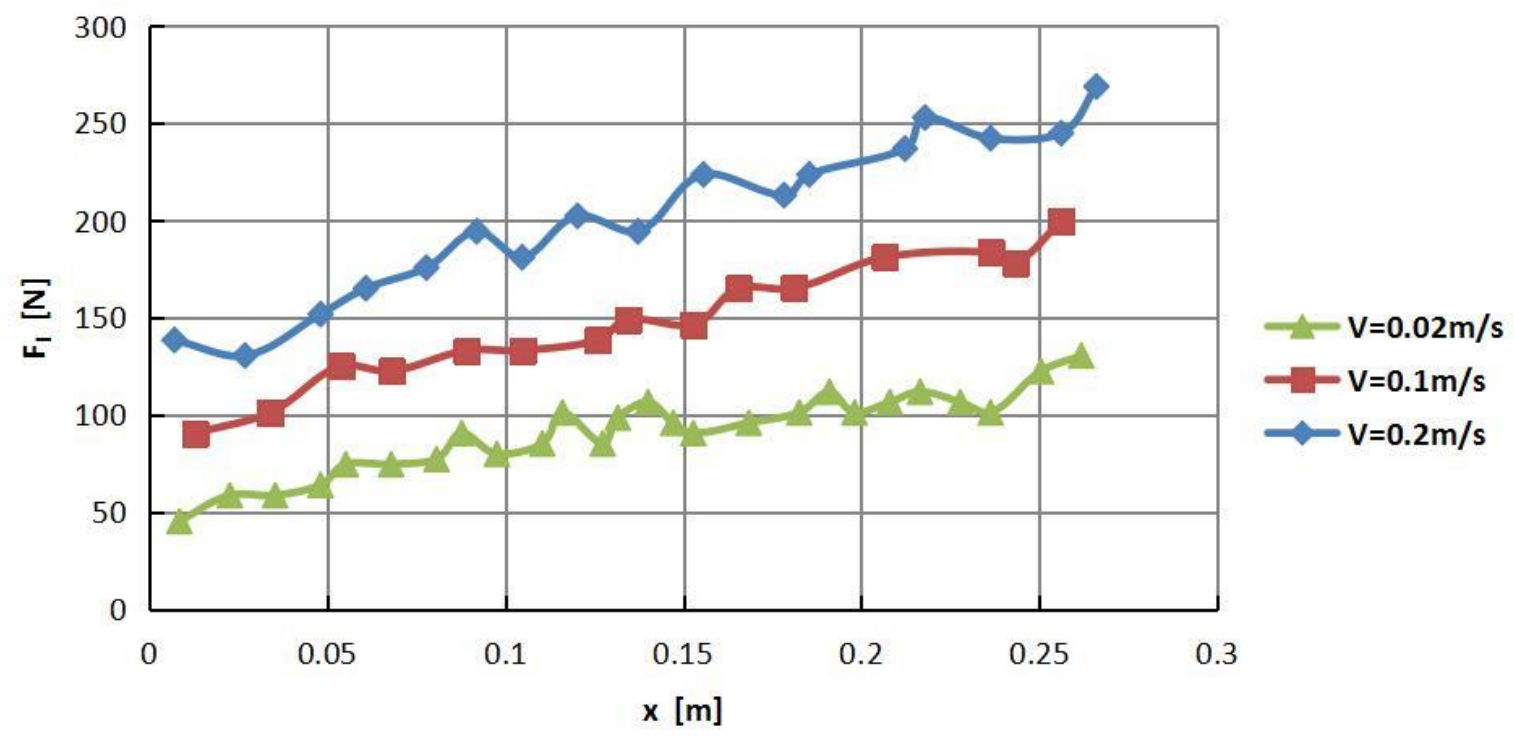

Figure 5(b)

Figure 5 | Velocity effect on (a) drag and (b) lift force when the inclined angle $\mathrm{A}$ is $60^{\circ}$ 
The effects of the inclination angle on drag and lift forces (drag velocity is $0.1 \mathrm{~m} / \mathrm{s}$ ) are described in figure 6. It shows that the drag force is almost independent of the inclination angle. However, the lift force exhibits completely different behaviour. When the immersion depth and drag velocity are both held constant, the drag force can maintain a constant value no matter how big the inclination angle is.

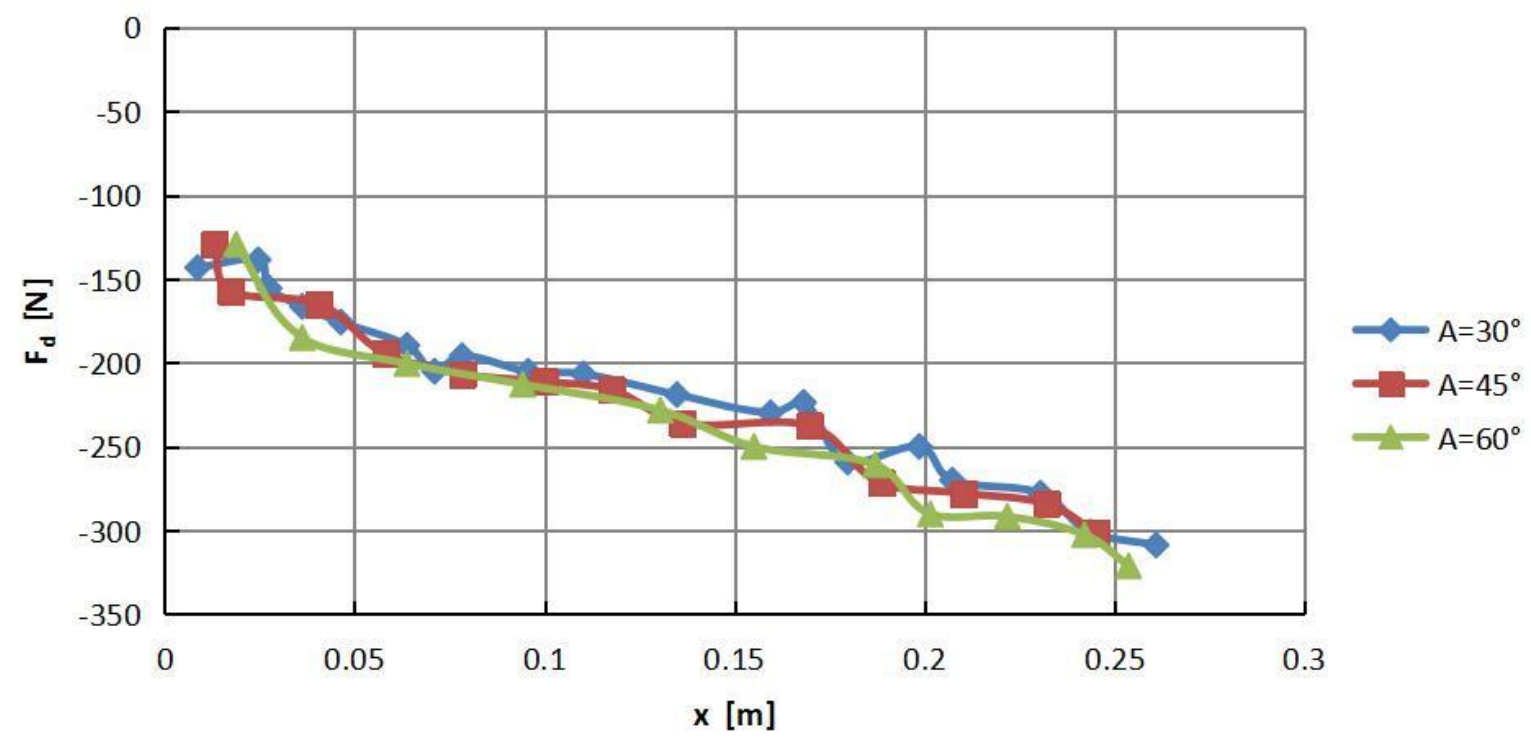

Figure 6(a)

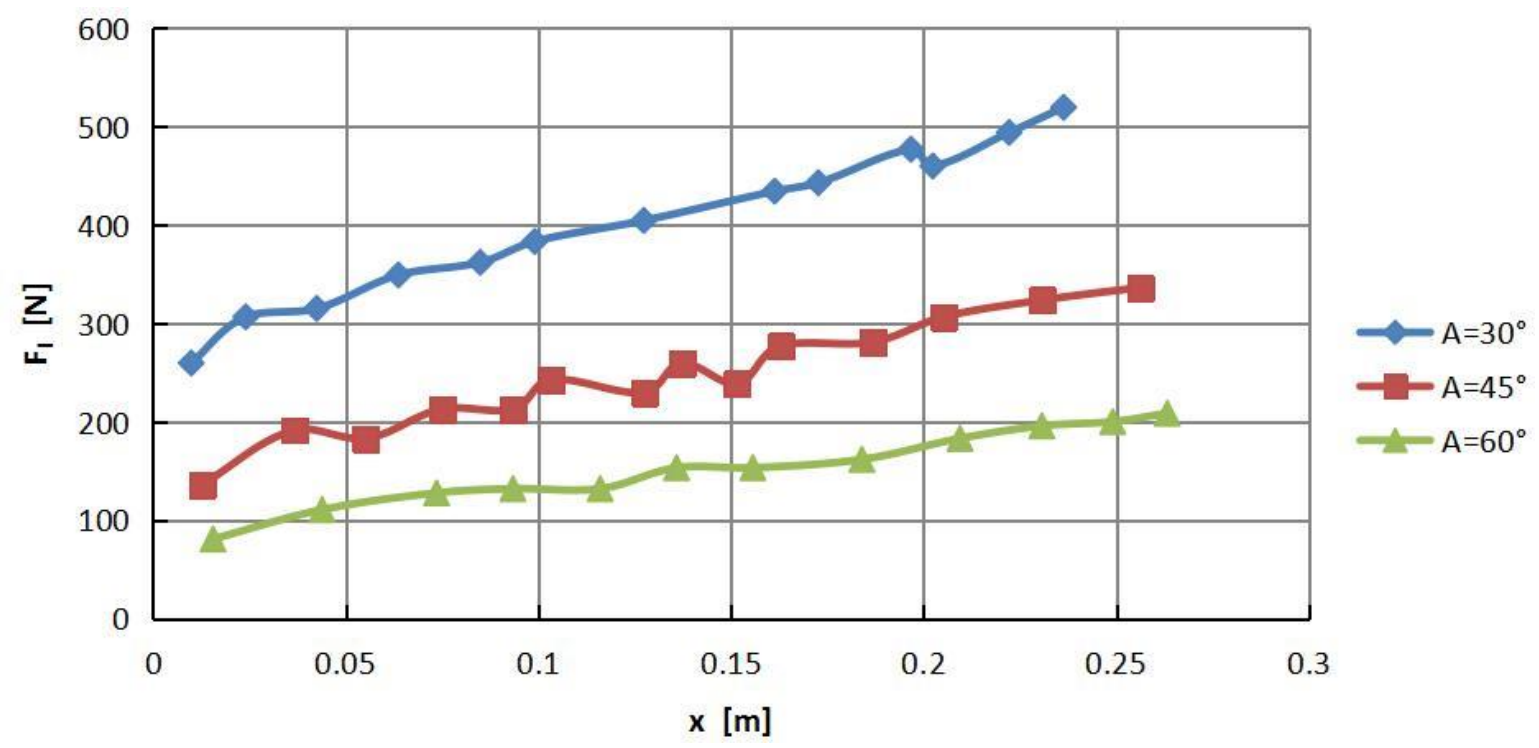

Figure 6(b)

Figure 6 | Effect of angle of attack on (a) drag and (b) lift force when the drag velocity is $0.1 \mathrm{~m} / \mathrm{s}$

The effects of velocity and inclination angle on the drag and lift forces still need to be investigated. We found through DEM simulation that the ratio of drag and lift force is equal to the tangent value of the inclination angle (see figure 7). 


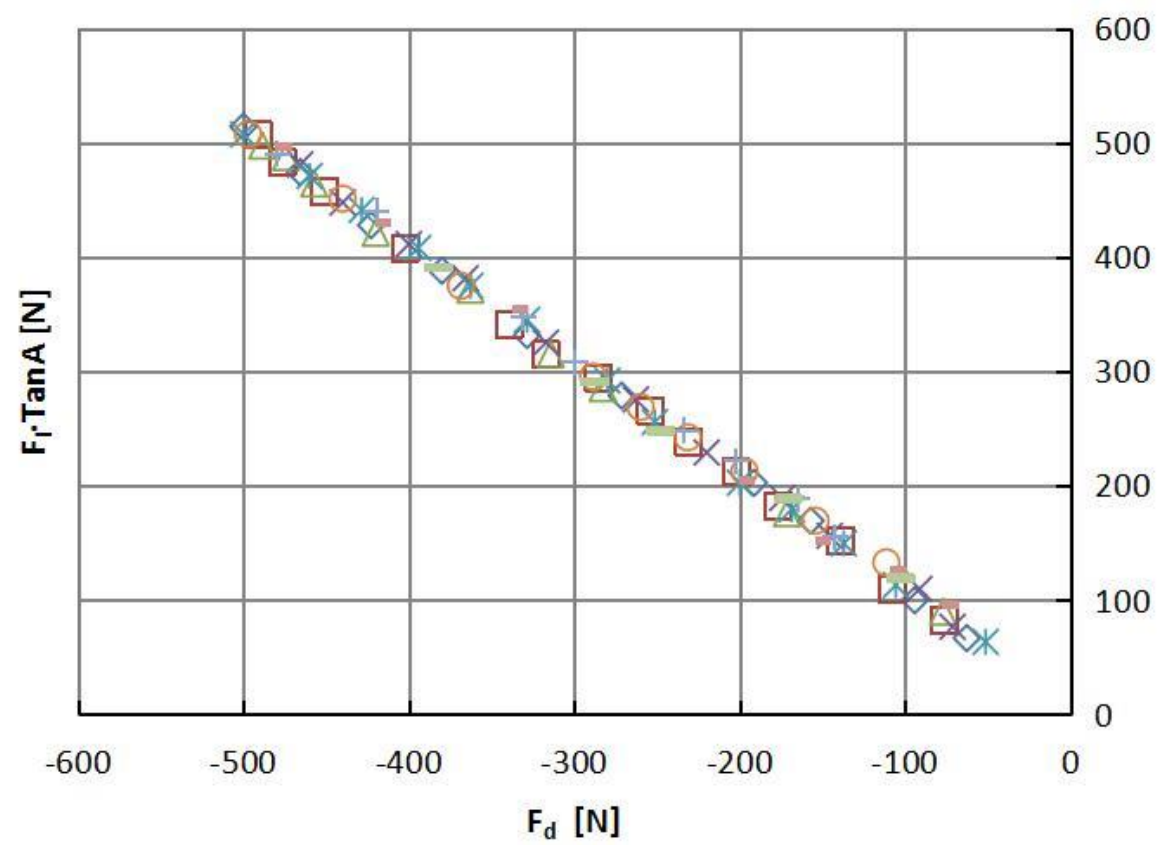

$\diamond \mathrm{V}=0.2 \mathrm{~m} / \mathrm{s}, \mathrm{A}=30^{\circ}$

$\square \mathrm{V}=0.1 \mathrm{~m} / \mathrm{s}, \mathrm{A}=30^{\circ}$

$\triangle \mathrm{V}=0.02 \mathrm{~m} / \mathrm{s}, \mathrm{A}=30^{\circ}$

$\times V=0.2 \mathrm{~m} / \mathrm{s}, A=45^{\circ}$

$* \mathrm{~V}=0.1 \mathrm{~m} / \mathrm{s}, \mathrm{A}=45^{\circ}$

$V=0.02 \mathrm{~m} / \mathrm{s}, A=45^{\circ}$

$+V=0.2 \mathrm{~m} / \mathrm{s}, A=60^{\circ}$

$=\mathrm{V}=0.1 \mathrm{~m} / \mathrm{s}, \mathrm{A}=60^{\circ}$

$-V=0.02 \mathrm{~m} / \mathrm{s}, A=60^{\circ}$

Figure 7 | Relation of $F_{d}$ and $F_{l} \tan A$

Next, we describe the drag and lift forces in a continuous way. From the velocity field we can see that there is a rather clear shear band from the bottom of the rigid body to the granular bed surface. Based on our simulations, we have found that the angle of repose remains constant. For a certain displacement and inclination angle, the total area of the triangle (or the mass) in front of the plate is the same no matter how large the speed is. It is rather difficult to judge in this case where the exact position of the shear band is. Here we propose a special way to deal with this: we assume that the shear band is clear enough, so in a certain displacement $x$, the total area (mass) is certain and the shape of the wedge remains constant. Therefore, the only difference should be the frictional property in the shear band. Based on this concept, we propose a dynamic friction coefficient, which is equal to the tangent value of the dynamic friction angle .

The reason we use this dynamic friction coefficient is that the flow induced by the inclined plate is not quasi-static. In references[14-15], they showed that the effective friction coefficient depends on the inertial number, $\mu_{\text {effective }}=\mu(I)$. The inertial number[16], which can quantify the flow regime,

can be expressed as: $I=\frac{\dot{\gamma} d}{\sqrt{P / \rho_{d}}}$, where $\dot{\gamma}$ is the shear rate, $d$ is the grain diameter, $P$ is the confining pressure and $\rho_{d}$ is the grain density. Figure 8 shows the distribution of inertial number when $x=$ $6 d$ in three different cases: (a) $V=0.2 \mathrm{~m} / \mathrm{s}, A=60^{\circ}$; (b) $V=0.1 \mathrm{~m} / \mathrm{s}, A=45^{\circ} ;$ (c) $V=0.02 \mathrm{~m} / \mathrm{s}, A=30^{\circ}$. It is rather clear that inertial number is roughly larger than $10^{-3}$ (dense flow) nearby the plate and smaller than $10^{-3}$ (quasi-static) in the majority of the regions. Generally, the inertial number near the wedge increases with the drag velocity. Since the inertial number varies in the different regions and for different time steps, the flow regime is rather complicated. Therefore, it is not easy to capture how the inertial number changes in the assumed shear band. This is why we use a variable friction coefficient, termed the "dynamic friction coefficient," to simplify this complicated friction property in the assumed shear band. The dynamic friction angles in different cases are shown in table 1. 


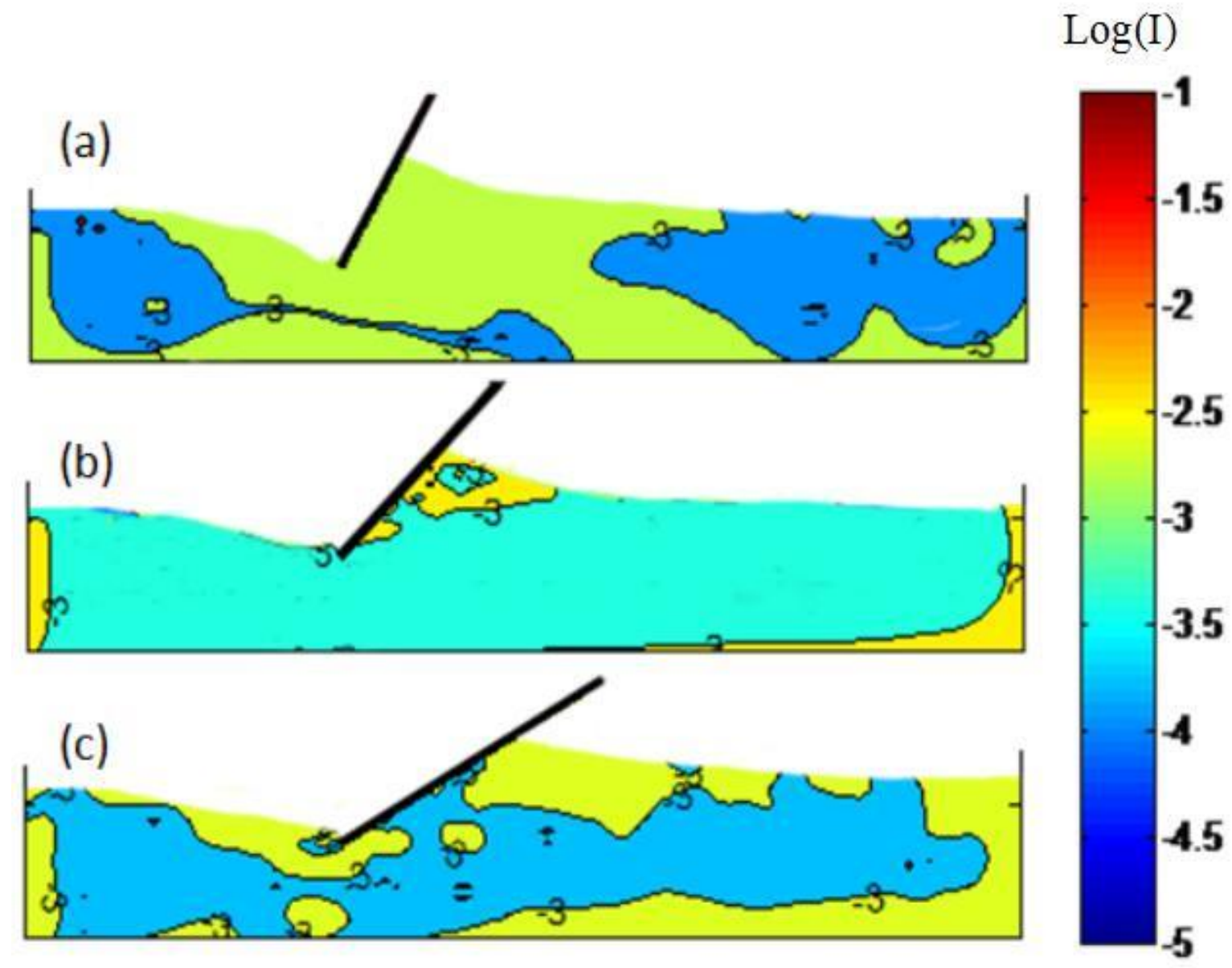

Figure 8 | Inertial number distribution when $x=6 d$

Table 1 Dynamic friction angle (in degrees) under different inclination angles and drag velocities

\begin{tabular}{cccc}
\hline & $A=30^{\circ}$ & $A=45^{\circ}$ & $A=60^{\circ}$ \\
\hline$V=0.2 \mathrm{~m} / \mathrm{s}$ & 10 & 16 & 20 \\
$V=0.1 \mathrm{~m} / \mathrm{s}$ & 8 & 12 & 15 \\
$V=0.02 \mathrm{~m} / \mathrm{s}$ & 5 & 6 & 8 \\
\hline
\end{tabular}

As is shown in figure 9, the displacement of the inclined plate is $x$, the pile-up height is $h$, the angle of repose is $\alpha_{r}$, the angle of the shear band is $\theta$, immersion depth is $Z$, and $C$ is the clearance. 


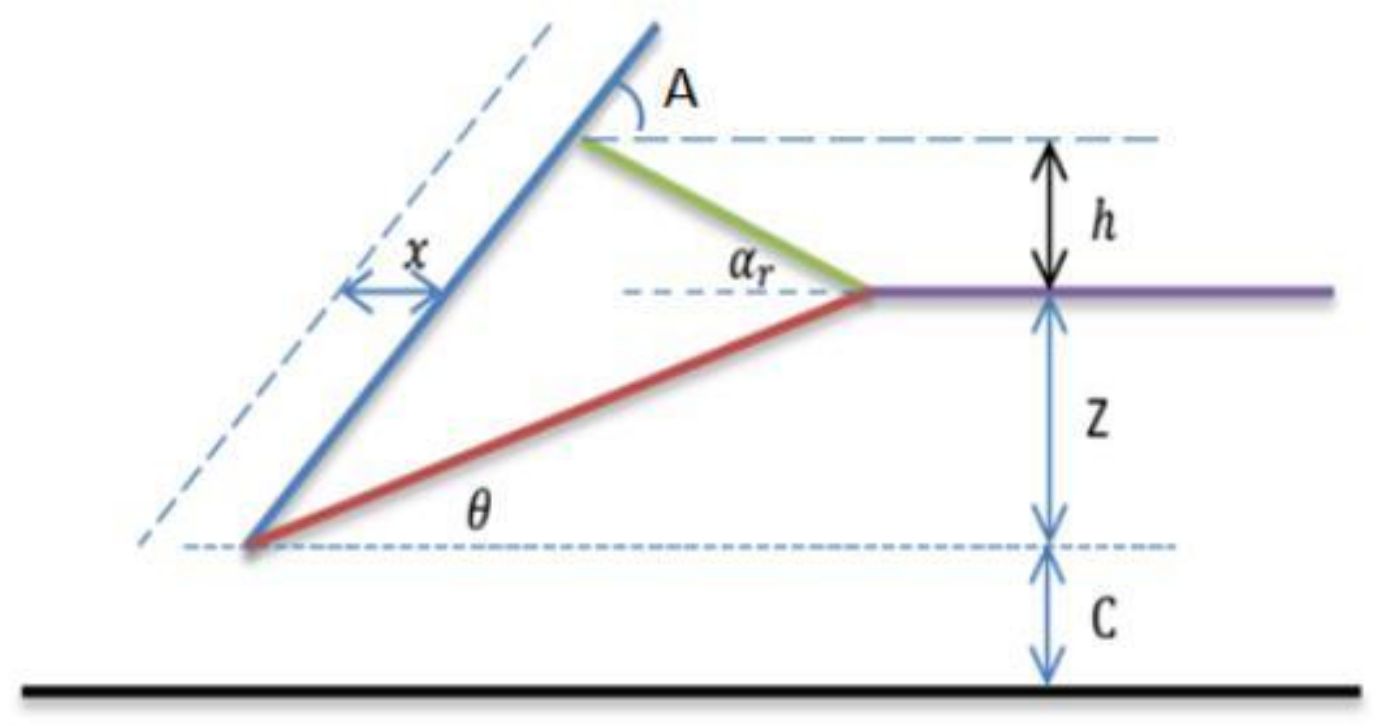

Figure 9 | Schematic side view of the model

We calculate the pile-up height, which is

$$
h=\sqrt{2 x Z /\left(1 / \tan A+1 / \tan \alpha_{r}\right)} .
$$

The area of the triangle (wedge) is

$$
S=h / 2 \cdot(h+Z)\left(1 / \tan A+1 / \tan \alpha_{r}\right) .
$$

The tangent value of $\theta$ is

$$
\tan \theta=Z /\left[h\left(1 / \tan A+1 / \tan \alpha_{r}\right)+Z / \tan A\right] .
$$

Hence, we obtain the total force applied on the inclined plate, which is

$$
F=\frac{S \rho^{*}\left(\mu_{d} \cos \theta+\sin \theta\right)}{\sin A\left(\cos \theta+\mu_{d} \sin \theta\right)-\cos A\left(\mu_{d} \cos \theta+\sin \theta\right)} .
$$

Therefore, drag and lift force can be described as follows

$$
\begin{aligned}
& F_{d}=F \sin A \\
& F_{l}=F \cos A
\end{aligned}
$$

The ratio of drag and lift forces in reference ${ }^{[15]}$ is slightly larger than that in this paper, it is likely because that the differences in the frictional properties of plate surfaces.

The comparison of the predicted and simulated values of the pile-up height and total force when $V$ $=0.1 \mathrm{~m} / \mathrm{s}, A=45^{\circ}$ are shown in figures $10(\mathrm{a})$ and (b), respectively. It can be seen that the model can predict the total force very well. The dynamic frictional angle $\phi_{d}$ is the only variable that is related to drag velocity and angle of attack. Specifically, the total force increases with both drag velocity and angle of attack due to the growing $\phi_{d}$. 


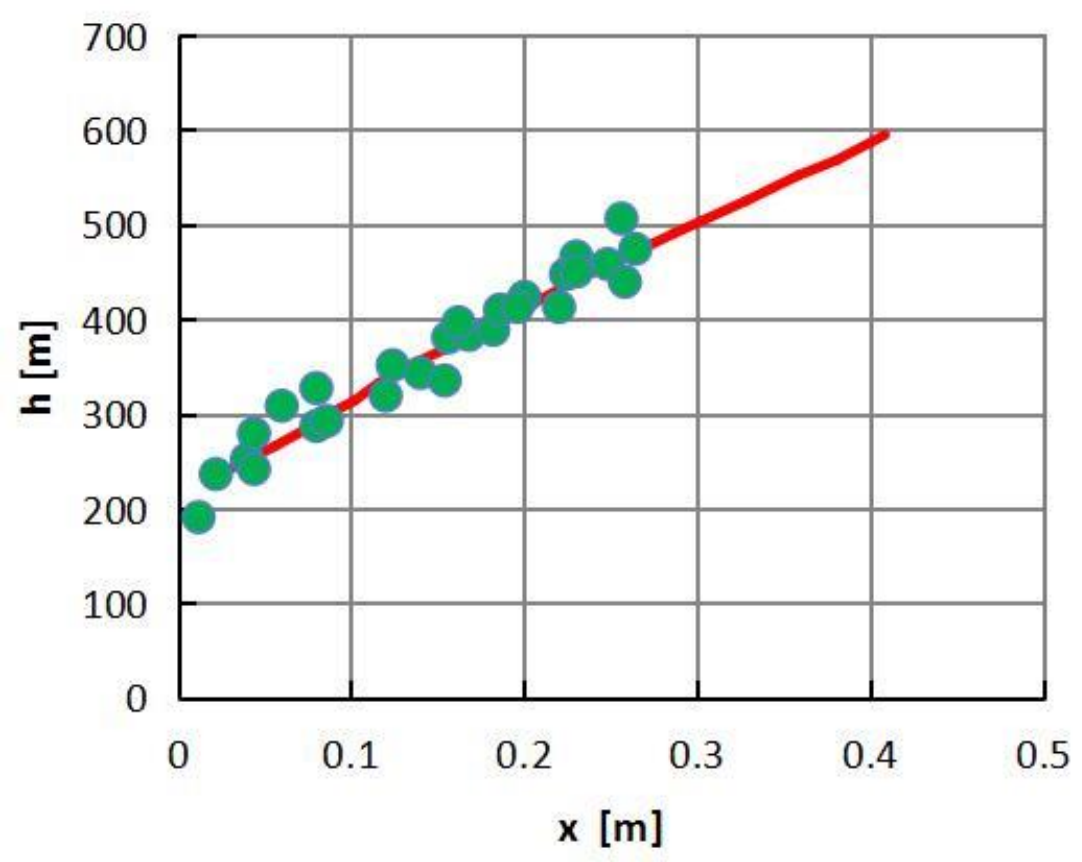

simulated values

predicted values

Figure 10(a)

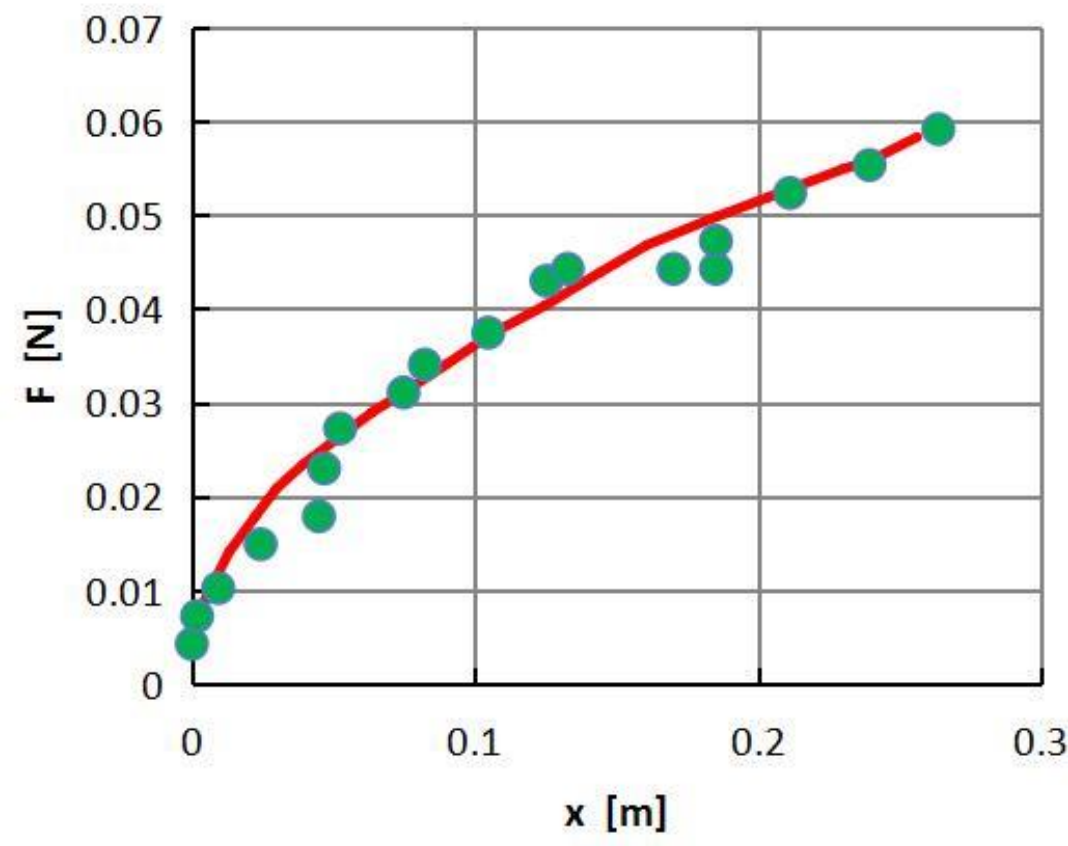

simulated values

predicted values

Figure 10(b)

Figure 10 I (a)Simulated values and predicted values for pile-up height, (b) Simulated values and predicted values for the total force acting on the plate; $\left(V=0.1 \mathrm{~m} / \mathrm{s}, A=45^{\circ}\right)$.

\section{Conclusions}

In this paper, we have extended the traditional Coulomb passive earth pressure model. For this inclined plate dragging problem, we have found that the drag and lift forces are the vertical and horizontal components of the total force acting on the inclined plate, and this is in good agreement 
with the results from the literature. In addition, we have proposed a dynamic friction angle and dynamic friction coefficient, which describe the complicated friction surface (shear band) very well. The advantage of this model is that it just has one variable-dynamic friction angle, which can be obtained by the parameters fitted in experiments or simulations, or empirically. Further work for our study may include the inner mechanics for this complicated frictional property in shear bands.

Author Contributions: For research articles with several authors, a short paragraph specifying their individual contributions must be provided. The following statements should be used "conceptualization, Hong Guo; methodology, Hong Guo; software, Hong Jiang; validation, Hong Guo; formal analysis, Rui Guo; resources, Nengyuan Chen; data curation, Jiangtao Fu.; writing-original draft preparation, Hong Guo; writing-review and editing, Hong Guo, Rui Guo, Hong Jiang, Jiangtao Fu."

Funding: This research was funded by National Natural Science Foundation of China, grant number 11702163, Shaanxi Province 2018 Key R\&D Program, grant number 2018ZDXM-SF-024 and Research Foundation for Talented Scholars of Shaanxi University of Technology, grant number SLGQD2017-03.

Acknowledgments: I would like to express my gratitude to all those who have helped me during the writing of the paper. Especially, I gratefully acknowledge the financial support of the National Natural Science Foundation of China.

Conflicts of Interest: The authors declared that they have no conflicts of interest to this work. We declare that we do not have any commercial or associative interest that represents a conflict of interest in connection with the work submitted

\section{References}

1. Goldsmith, J., Guo, H., Hunt, S. N., Tao, M., \& Koehler, S. (2013). Drag on intruders in granular beds: A boundary layer approach. Physical Review E, 88(3), 030201.

2. Guo, H., Goldsmith, J., Delacruz, I., Tao, M., Luo, Y., \& Koehler, S. A. (2012). Semi-infinite plates dragged through granular beds. Journal of Statistical Mechanics: Theory and Experiment, 2012(07), P07013.

3. Fang, Y. S., Chen, T. J., \& Wu, B. F. (1994). Passive earth pressures with various wall movements. Journal of Geotechnical Engineering, 120(8), 1307-1323.

4. Percier, B., Manneville, S., McElwaine, J. N., Morris, S. W., \& Taberlet, N. (2011). Lift and drag forces on an inclined plow moving over a granular surface. Physical Review E, 84(5), 051302.

5. Percier, B., Manneville, S., \& Taberlet, N. (2013). Modeling a washboard road: From experimental measurements to linear stability analysis. Physical Review E, 87(1), 012203.

6. Taberlet, N., Morris, S. W., \& McElwaine, J. N. (2007). Washboard road: the dynamics of granular ripples formed by rolling wheels. Physical review letters, 99(6), 068003.

7. Goldman, D. I., \& Umbanhowar, P. (2008). Scaling and dynamics of sphere and disk impact into granular media. Physical Review E, 77(2), 021308.

8. Bitbol, A. F., Taberlet, N., Morris, S. W., \& McElwaine, J. N. (2009). Scaling and dynamics of washboard roads. Physical Review E, 79(6), 061308.

9. Hewitt, I. J., Balmforth, N. J., \& McElwaine, J. N. (2012). Granular and fluid washboards. Journal of Fluid Mechanics, 692, 446-463.

10. Mays, D. C., \& Faybishenko, B. A. (2000). Washboards in unpaved highways as a complex dynamic system. Complexity, 5(6), 51-60.

11. Both, J. A., Hong, D. C., \& Kurtze, D. A. (2001). Corrugation of roads. Physica A: Statistical Mechanics and its Applications, 301(1), 545-559.

12. Zhong, W. Z., He, K. J., Zhou, Z. Y., Xia, W., \& Li, Y. Y. (2009). Calibration of the damping coefficient for the DEM simulation. Aeta Physica Sinica, 58(8), 5155-5161.

13. Di-Ping, W., Xing-Xiang, L., Qin, Q., Ben, G., \& Yong, Z. (2014). Study on mechanical behavior of the transverse processing on a granular matter layer.17. P. Jop, Y. Forterre, O. Pouliquen, Nature 441, 727(2006).

14. Forterre, Y., \& Pouliquen, O. (2008). Flows of dense granular media. Annu. Rev. Fluid Mech., 40, 1-24.

15. Maladen, R. D., Umbanhowar, P. B., Ding, Y., Masse, A., \& Goldman, D. I. (2011, May). Granular lift forces predict vertical motion of a sand-swimming robot. In Robotics and Automation (ICRA), 2011 IEEE International Conference on (pp. 1398-1403). IEEE. 
16. Trulsson, M., DeGiuli, E., \& Wyart, M. (2017). Effect of friction on dense suspension flows of hard particles. Physical Review E, 95(1), 012605. 